\title{
DESEMPENHO DE MUDAS DE MANJERICÃO CV. MARIA BONITA SUBMETIDAS A PROPORÇÕES DE NITRATO E AMÔNIO
}

\author{
Lavine Silva Matos ${ }^{1}$; Gilvanda Leão dos Anjos ${ }^{2}$, Diego dos Santos Souza ${ }^{2}$, \\ Nalbert Silva dos Santos ${ }^{2}$ e Anacleto Ranulfo dos Santos ${ }^{3}$.
}

1. Pós-graduanda em Solos e Qualidade de Ecossistemas da Universidade Federal do Recôncavo da Bahia (lavinematos@yahoo.com.br). Cruz das Almas - Brasil.

2. Graduando (a) em Engenharia Agronômica da Universidade Federal do Recôncavo da Bahia.

3. Professor Doutor do Centro de Ciências Agrárias, Ambientais e Biológicas da Universidade Federal do Recôncavo da Bahia.

Recebido em: 08/09/2015 - Aprovado em: 14/11/2015 - Publicado em: 01/12/2015 DOI: http://dx.doi.org/10.18677/Enciclopedia_Biosfera_2015_125

\begin{abstract}
RESUMO
O objetivo desse trabalho foi avaliar o desempenho de mudas de manjericão cv. Maria Bonita submetidas a proporções dos íons $\mathrm{NO}_{3}{ }^{-}$e $\mathrm{NH}_{4}{ }^{+}$quanto ao seu crescimento inicial e índices de clorofila. O experimento foi conduzido sob delineamento experimental inteiramente casualizado (DIC), com cinco repetições, em que cada parcela experimental foi constituída de uma planta. Os tratamentos consistiram na solução de HOAGLAND \& ARNON (1950), modificada devido às diferentes proporções $\left(\mathrm{NO}_{3}{ }^{-}: \mathrm{NH}_{4}{ }^{+}\right): 100: 0 ; 75: 25 ; 50: 50 ; 25: 75 ; 0: 100$. Verificou-se que o suprimento isolado de amônio (0:100) proporcionou as menores médias das variáveis avaliadas. As variáveis massa seca das folhas, hastes, parte aérea e massa seca total foram favorecidas pelos tratamentos correspondentes as proporções 100:0, 75:25 e 50:50 de nitrato e amônio, enquanto que a massa seca da raiz e a relação raiz/parte aérea obtiveram seus maiores valores no tratamento com omissão de amônio (100:0). Os maiores valores de clorofila $a, b$ e total $(a+b)$ foram obtidos com a proporção 50:50 de nitrato e amônio.
\end{abstract}

PALAVRAS-CHAVE: interação iônica, Ocimum basilicum L., formas nitrogenadas.

\section{PERFORMANCE OF BASIL PLANTS CV. MARIA BONITA UNDER PROPORTIONS NITRATE AND AMMONIUM}

\begin{abstract}
The objective of this study was to evaluate the performance of basil seedlings $\mathrm{cv}$. Maria Bonita submitted the proportions of NO3- and $\mathrm{NH} 4+$ ions as its initial growth and chlorophyll contents. The experiment was conducted in a completely randomized design (CRD) with five replications, each experimental plot consisted of a plant. Treatments consisted in solving HOAGLAND \& ARNON (1950), modified due to different proportions (NO3-: $\mathrm{NH}_{4}+$ ): 100:0; 75:25; 50:50; 25:75; 0:100. It was found that the isolated supply of ammonium (0:100) provided the lowest average of variables. The variables dry mass of leaves, stems, shoots and total dry mass were favored by the corresponding treatments ratios 100: 0, 75:25 and 50:50 nitrate and ammonium, while root dry mass and root/shoot obtained their highest values at ENCICLOPÉDIA BIOSFERA, Centro Científico Conhecer - Goiânia, v.11 n.22; p.888 2015
\end{abstract}


treatment with ammonium omission (100:0). The major chlorophyll values $a, b$ and total $(a+b)$ were obtained with 50:50 and ammonium nitrate.

KEYWORDS: ionic interaction, Ocimum basilicum L., nitrogen forms.

\section{INTRODUÇÃO}

O gênero Ocimum, originário do sudeste asiático e África Central, compreendem em torno de 3200 espécies que se adaptaram muito bem aos solos brasileiros (BLANK et al. 2010). O manjericão (Ocimum basilicum L.), pertencente à família Lamiaceae, é uma planta anual ou perene, dependendo do local em que é cultivado. No Brasil, é cultivado principalmente por pequenos produtores rurais para a comercialização da planta como condimento (TEIXEIRA et al., 2002). Além de seu uso in natura, é utilizado para obtenção de óleo essencial, muito importante na indústria de perfumaria, cosmético, medicamento e alimento (BLANK et al., 2007).

Um dos fatores mais importantes para obter altas produções em todas as culturas, é o suprimento de nitrogênio (ALVES et al. 2013). Ele representa de 1 a $6 \%$ da sua matéria seca das plantas. $\mathrm{O} \mathrm{NO}_{3}{ }^{-}$e $\mathrm{NH}_{4}{ }^{+}$são as formas de $\mathrm{N}$ preferencialmente absorvido e metabolizado pelas plantas e o que está mais abundante na solução do solo (RIBEIRO et al. 2012).

Comumente, plantas que se desenvolvem em solos com $\mathrm{pH}$ ácido preferem o $\mathrm{NH}_{4}{ }^{+}$como fonte de $\mathrm{N}$, enquanto plantas que se desenvolvem em solos com pH neutro a alcalino têm leve tendência a preferir o $\mathrm{NO}_{3}^{-}$(GUIMARÃES et al., 2014). Ainda segundo esses autores, a assimilação de N, por dispensar as fases de redução demanda menor quantidade de energia, quando absorvido sob a forma de $\mathrm{NH}_{4}{ }^{+}$, energia, que e requeridas quando ocorre absorção de $\mathrm{NO}_{3}{ }^{-}$. Porém em alta concentração, o $\mathrm{NH}_{4}{ }^{+}$pode ser citotóxico, causando clorose, além da diminuição do crescimento, se comparado ao $\mathrm{NO}_{3}{ }^{-} \mathrm{em}$ igual concentração.

A absorção radicular de $\mathrm{NH}_{4}{ }^{+}$, mediada por um transportador, depende de sua única entrada, e quando ele é levado para o citoplasma da célula, ocasiona um desequilíbrio eletrostático entre o meio intra e extracelular, fazendo com que a célula promova um fluxo contrário de cargas positivas, a fim de alcançar a neutralidade e, em meio nutritivo, o pH apresenta dupla importância: influi na forma iônica de vários elementos, no equilíbrio oxidação-redução e na solubilidade de vários constituintes, também influi nas raízes das plantas, especialmente na membrana transportadoras de íons na epiderme e córtex da raiz (SILVA et al., 2010).

Levando em conta que as plantas apresentam múltiplas respostas às formas nitrogenadas, nítrica e amoniacal, este estudo teve por objetivo avaliar o crescimento inicial e índices de clorofila de mudas de manjericão cv. Maria Bonita submetidas a proporções dos íons $\mathrm{NO}_{3}{ }^{-}$e $\mathrm{NH}_{4}{ }^{+}$.

\section{MATERIAL E MÉTODOS}

O experimento foi conduzido na Universidade Federal do Recôncavo da Bahia - UFRB, em Cruz das Almas, no período de junho a agosto de 2015. As sementes de manjericão foram germinadas em sementeiras com 72 células. Nesse período as plântulas foram irrigadas diariamente com solução nutritiva de HOAGLAND \& ARNON (1950) modificada (sem nitrogênio) e com 50\% da força iônica.

Após 21 dias as plântulas foram transplantadas para recipientes plásticos contendo $2 \mathrm{dm}^{3}$ de areia lavada, onde permaneceram até o final do experimento. As plantas foram cultivadas por 40 dias em condições de casa de vegetação. $O$ experimento foi conduzido sob delineamento experimental inteiramente casualizado 
(DIC), com cinco repetições, e cada parcela experimental foi constituída de uma planta. A solução nutritiva de HOAGLAND \& ARNON (1950) modificada utilizada no experimento contendo as proporções de nitrato $\left(\mathrm{NO}_{3}{ }^{-}\right)$e amônio $\left(\mathrm{NH}_{4}{ }^{+}\right)$está descrita na Tabela 1.

Ao final do experimento foram avaliadas as seguintes variáveis: altura da planta (AP), diâmetro da haste (DH), comprimento da raiz (CR), número de folhas (NF), e índices de clorofila. Para a determinação da altura da planta e do comprimento das raízes, foi utilizada uma régua graduada. $\mathrm{O}$ diâmetro da haste foi obtido por meio da utilização de um paquímetro digital, e o número de folhas foi determinado por meio de contagem direta. Os índices de clorofila (Índice de Clorofila Falker: ICF) foram obtidos por meio do medidor eletrônico de clorofila Falker (clorofiLOG) - modelo CFL1030.

Após a secagem em estufa de circulação de ar forçada $\left(65^{\circ} \mathrm{C}\right)$ por $72 \mathrm{~h}$, foram obtidos os valores de: massa seca de folhas, hastes, parte aérea, raízes e total (MSF, MSH, MSPA, MSR, MST) e relação raiz/parte aérea (R/PA). Estes valores foram encontrados utilizando-se balança de precisão com três casas. A Área Foliar (AF) foi determinada pelo método dos discos foliares por meio de formula matemática conforme PEIXOTO et al. (1998). A relação raiz/parte aérea foi encontrada segundo metodologia proposta por BENINCASA (1988).

Os resultados obtidos foram submetidos à análise de variância utilizando-se 0 programa estatístico SISVAR (FERREIRA, 2011). Em função do nível de significância será aplicado o teste de Tukey a $5 \%$ de probabilidade.

TABELA 1. Volumes $(\mathrm{mL})$ retirados das soluções estoque para formar $1 \mathrm{~L}$ de solução nutritiva modificada, seguindo os respectivos tratamentos com as doses de $\mathrm{NO}_{3}{ }^{-}: \mathrm{NH}_{4}{ }^{+}$. Cruz das Almas, 2015.

\begin{tabular}{cccccc}
\hline $\begin{array}{c}\text { Solução } \\
\text { estoque }\end{array}$ & \multicolumn{5}{c}{ Proporções $\left(\mathbf{N O}_{3} \mathbf{}^{-}: \mathbf{N H}_{4}{ }^{+}\right)$} \\
\cline { 2 - 6 }$(\mathbf{1 M})$ & $\mathbf{1 0 0 : 0}$ & $\mathbf{7 5 : 2 5}$ & $\mathbf{5 0 : 5 0}$ & $\mathbf{2 5 : 7 5}$ & $\mathbf{0 : 1 0 0}$ \\
\hline $\mathrm{KH}_{2} \mathrm{PO}_{4}$ & 1 & 1 & 1 & 1 & 1 \\
$\mathrm{NH}_{4} \mathrm{Cl}$ & - & 3,75 & 7,5 & 11,25 & 15 \\
$\mathrm{KCl}$ & - & 3,8 & - & 1,2 & 5 \\
$\mathrm{CaCl}$ & - & - & 3 & 5 & 5 \\
$\mathrm{MgSO}_{4}$ & 2 & 2 & 2 & 2 & 2 \\
$\mathrm{KNO}_{3}$ & 5 & 1,2 & 5 & 3,75 & - \\
$\mathrm{Ca}\left(\mathrm{NO}_{3}\right)_{2}$ & 5 & 5 & 2 & - & - \\
Ferro-EDTA & 1 & 1 & 1 & 1 & 1 \\
Micronutrientes & 1 & 1 & 1 & 1 & 1 \\
\hline
\end{tabular}

*Solução de Ferro-EDTA: Serão dissolvidos $26,1 \mathrm{~g}$ de EDTA dissódico em $286 \mathrm{~mL}$ de $\mathrm{NaOH} 1 \mathrm{~N}+24,9 \mathrm{~g}$ de $\mathrm{FeSO}_{4} .7 \mathrm{H}_{2} \mathrm{O}$ e aerado por uma noite.

${ }^{*}$ Solução de micronutrientes $(\mathrm{g} / \mathrm{l}): \mathrm{H}_{3} \mathrm{BO}_{3}=2,86 ; \mathrm{MnCl}_{2} .4 \mathrm{H}_{2} \mathrm{O}=1,81 ; \mathrm{ZnCl}_{2}$ $=0,10 ; \mathrm{CuCl}_{2}=0,04 ; \mathrm{H}_{2} \mathrm{MoO}_{4} \cdot \mathrm{H}_{2} \mathrm{O}=0,02$. 


\section{RESULTADOS E DISCUSSÃO}

Houve efeito significativo $(p<0,05)$ para todas as variáveis avaliadas, devido aos tratamentos utilizados, além disso, durante o período experimental foi possível observar sintomas de toxidez nas plantas que receberam as soluções nas seguintes proporções de $\mathrm{NO}_{3}{ }^{-}: \mathrm{NH}_{4}{ }^{+}:$25:75 e 0:100.

TABELA 2. Resumo da análise de variância com os $s$ quadrados médios para as variáveis de crescimento das plantas de manjericão cultivadas em diferentes proporções de NH4:NO3, aplicados em solução nutritiva.Cruz das Almas-BA, 2015.

\begin{tabular}{|c|c|c|c|c|c|c|c|c|c|c|c|c|c|c|c|c|}
\hline FV & AP & $\mathrm{DH}$ & NF & CR & $\begin{array}{c}\text { MS } \\
\text { F }\end{array}$ & $\begin{array}{c}\text { MS } \\
\text { H }\end{array}$ & $\begin{array}{l}\text { MS } \\
\text { PA }\end{array}$ & $\begin{array}{c}\text { MS } \\
\mathbf{R}\end{array}$ & $\begin{array}{c}\text { MS } \\
T\end{array}$ & $\begin{array}{l}\text { R/ } \\
\text { PA }\end{array}$ & $\mathbf{A F}$ & $\begin{array}{c}\text { AF } \\
E\end{array}$ & $\begin{array}{c}\text { RA } \\
\mathbf{F}\end{array}$ & CLa & $\begin{array}{c}C L \\
b\end{array}$ & $\begin{array}{c}\text { CL } \\
\text { Total } \\
(a+b)\end{array}$ \\
\hline & \multicolumn{16}{|c|}{ - QUADRADOS MÉDIOS } \\
\hline Trat & $\begin{array}{l}248, \\
79^{*}\end{array}$ & $\begin{array}{c}0,1 \\
5^{*}\end{array}$ & $\begin{array}{l}7319 \\
84^{*}\end{array}$ & $\begin{array}{c}360,7 \\
1^{*}\end{array}$ & $\begin{array}{l}3,6 \\
8^{*}\end{array}$ & $\begin{array}{l}0,9 \\
1^{*}\end{array}$ & $\underset{*}{7,92}$ & $6,4^{*}$ & $\begin{array}{c}26,8 \\
6^{*}\end{array}$ & $\begin{array}{c}0,3 \\
6^{*}\end{array}$ & $\begin{array}{c}0,8 \\
1^{*}\end{array}$ & $\begin{array}{l}16 \\
4^{*}\end{array}$ & $\underset{*}{1.85}$ & $\begin{array}{c}40,6 \\
4^{*}\end{array}$ & $\begin{array}{l}21 \\
8^{*}\end{array}$ & $34,3^{*}$ \\
\hline $\begin{array}{l}\text { Resí- } \\
\text { duo }\end{array}$ & $\begin{array}{c}15.3 \\
96\end{array}$ & $\begin{array}{l}0.0 \\
08\end{array}$ & $\begin{array}{c}300.8 \\
40\end{array}$ & $\begin{array}{c}21.03 \\
8\end{array}$ & $\begin{array}{l}0.1 \\
18\end{array}$ & $\begin{array}{l}0.0 \\
49\end{array}$ & $\begin{array}{c}0.30 \\
0\end{array}$ & $\begin{array}{l}0.5 \\
56\end{array}$ & $\begin{array}{c}1.30 \\
2\end{array}$ & $\begin{array}{l}0.0 \\
47\end{array}$ & $\begin{array}{l}0.0 \\
76\end{array}$ & $\begin{array}{l}0.4 \\
02\end{array}$ & $\begin{array}{c}0.01 \\
02\end{array}$ & $\begin{array}{c}4.01 \\
5\end{array}$ & $\begin{array}{l}1.7 \\
39\end{array}$ & 10.376 \\
\hline CV (\%) & $\begin{array}{c}20,9 \\
9\end{array}$ & $\begin{array}{l}17, \\
75\end{array}$ & 24,98 & 20,26 & $\begin{array}{l}21 \\
79\end{array}$ & $\begin{array}{c}36 \\
1\end{array}$ & $\begin{array}{c}25,0 \\
1\end{array}$ & $\begin{array}{l}56 \\
22\end{array}$ & $\begin{array}{c}32,4 \\
4\end{array}$ & $\begin{array}{l}43 \\
37\end{array}$ & $\begin{array}{l}28 \\
32\end{array}$ & $\begin{array}{c}58, \\
2\end{array}$ & $\begin{array}{c}18.3 \\
3\end{array}$ & 8,13 & $\begin{array}{c}16 \\
8\end{array}$ & 9,9 \\
\hline $\begin{array}{l}\text { Media } \\
\text { geral }\end{array}$ & $\begin{array}{c}18,6 \\
9\end{array}$ & $\begin{array}{c}0,5 \\
0\end{array}$ & 69,44 & 22,64 & $\begin{array}{c}1,5 \\
8\end{array}$ & $\begin{array}{c}0,6 \\
1\end{array}$ & 2,19 & $\begin{array}{c}1,3 \\
3\end{array}$ & 3,52 & $\begin{array}{c}0,5 \\
0\end{array}$ & $\begin{array}{c}0,9 \\
7\end{array}$ & 1,1 & 0.55 & $\begin{array}{c}24,6 \\
6\end{array}$ & 7,8 & 32,5 \\
\hline \multicolumn{17}{|c|}{$\begin{array}{l}\text { AP - altura da planta (cm); DH - diâmetro da haste }(\mathrm{cm}) \text {; NF - número de folhas; CR - } \\
\text { comprimento de raiz; MSF, MSH, MSPA, MSR, MST - massa seca das folhas, hastes, } \\
\text { parte aérea, raiz e massa seca total; R/PA - relação raiz/parte aérea, AF - área foliar, } \\
\text { AFE - área foliar específica, RAF - razão de área foliar; CLOROFILA - } a, b \text { e T }(a+b) \text {; } \\
\text { - significativo ao nível de } 5 \% \text { pelo teste F. }\end{array}$} \\
\hline
\end{tabular}

Quanto a altura da planta verificou-se que as mudas submetidas as proporções 100:0, 75:25, 50:50 e 23:75 $\left(\mathrm{NO}_{3}{ }^{-}: \mathrm{NH}_{4}{ }^{+}\right)$não diferiram estatisticamente entre si, porém apresentaram médias superiores a dose de 0:100 (Tabela 2). Para as variáveis diâmetro da haste e comprimento de raiz, o mesmo ocorreu, tendo a proporção 0:100 sido responsável pelas menores médias. As proporções nitrato: amônio que proporcionaram os maiores números de folhas foram 100:0 e 75:25.

TABELA 2. Valores médios das variáveis altura da planta (AP), diâmetro da haste (DH), cumprimento da raiz (CR) e número de folhas (NF) das plantas de manjericão submetidas a diferentes proporções de $\mathrm{NO}_{3}{ }^{-}: \mathrm{NH}_{4}{ }^{+}$em solução nutritiva. Cruz das Almas, 2015.

\begin{tabular}{ccccc}
\hline $\begin{array}{c}\text { Relação } \\
\mathbf{N O}_{3}{ }^{-}: \mathbf{N H}_{4}{ }^{+}\end{array}$ & AP & DH & $\mathbf{C R}$ & NF \\
\hline $100: 0$ & $23,80 \mathrm{a}$ & $0,58 \mathrm{a}$ & $29,40 \mathrm{a}$ & $93,00 \mathrm{a}$ \\
$75: 25$ & $22,80 \mathrm{a}$ & $0,60 \mathrm{a}$ & $26,40 \mathrm{a}$ & $106,60 \mathrm{a}$ \\
$50: 50$ & $21,50 \mathrm{a}$ & $0,61 \mathrm{a}$ & $27,78 \mathrm{a}$ & $83,60 \mathrm{ab}$ \\
$25: 75$ & $18.84 \mathrm{a}$ & $0,52 \mathrm{a}$ & $21,10 \mathrm{a}$ & $53,40 \mathrm{~b}$ \\
$0: 100$ & $6.52 \mathrm{~b}$ & $0,20 \mathrm{~b}$ & $8,50 \mathrm{~b}$ & $10,60 \mathrm{c}$ \\
\hline CV $(\%)$ & 20,99 & 17,75 & 20,26 & 24,98 \\
\hline
\end{tabular}

Médias seguidas pelas mesmas letras minúsculas nas colunas não diferem estatisticamente entre si pelo teste de Tukey a 5\% de probabilidade. 
Estudando proporções de nitrato e amônio no cultivo de Eucalyptus urophylla, GUIMARÃES et al. (2014), observaram que o crescimento em altura foi significativamente influenciado pela proporção $75 / 25$ de $\mathrm{NO}_{3}{ }^{-}$e $\mathrm{NH}_{4}{ }^{+}$, por outro lado, as variáveis o diâmetro do colo, o número de folhas, a área foliar e a relação parte aérea/raiz não foram afetadas pelas proporções de $\mathrm{NO}_{3}{ }^{-}$e $\mathrm{NH}_{4}{ }^{+}$utilizadas no trabalho.

Ao estudarem o efeito do nitrato e amônio sobre o crescimento da berinjela, SOUSA et al. (2010), constataram que as plantas responderam significativamente onde a maior altura da planta foi obtida na relação nitrato: amônio de 75:25, assim como, o número de folhas foi afetado significativamente pelas relações nitrato:amônio, sendo observada resposta crescente até a relação 75:25.

Por outro lado, RIBEIRO et al. (2012) não obtiveram efeitos significativos dos tratamentos para comprimento da parte aérea e para diâmetro do caule, no entanto, foram observadas respostas significativas para o comprimento de raiz, massas seca da parte aérea e da raiz e número de folhas, demonstrando- se, assim, que a cultura do amendoinzeiro responde às diferentes relações entre os íons amônio e nitrato $\left(\mathrm{NO}_{3}{ }^{-}\right.$e $\left.\mathrm{NH}_{4}{ }^{+}\right)$na solução nutritiva.

Em relação à massa seca de folhas, hastes, parte aérea e massa seca total (Tabela 3), os maiores valores médios foram devido às proporções 100:0, 75:25 e $50: 50\left(\mathrm{NO}_{3}: \mathrm{NH}_{4}{ }^{+}\right)$, estas não diferiram estatisticamente entre si, mas mostraram-se superiores as demais proporções. A massa seca da raiz e a elação raiz/parte aérea foram favorecidas pela proporção 100:0, tendo todas as outras proporções valores inferiores.

Em estudo com girassol submetido a proporções de amônio e nitrato, ALVES et al. (2013) constataram que, quanto a massa seca da haste, verificou-se que as mudas submetidas às relações 0:100 e 25:75 $\left(\mathrm{NH}_{4}{ }^{+}: \mathrm{NO}_{3}{ }^{-}\right)$apresentaram maiores médias, e em relação aos teores de massa seca na folha e parte aérea, a presença do amônio provocou a redução destes parâmetros.

TABELA 3. Valores médios das variáveis massa seca das folhas, hastes parte aérea, raiz e total (MSF, MSH, MSPA, MSR, MST) e relação raiz/parte aérea $(\mathrm{R} / \mathrm{PA})$ das plantas de manjericão submetidas a diferentes proporções de $\mathrm{NO}_{3}{ }^{-}: \mathrm{NH}_{4}{ }^{+}$em solução nutritiva. Cruz das Almas, 2015.

\begin{tabular}{ccccccc}
\hline $\begin{array}{c}\text { Relação } \\
\mathbf{N O}_{3}^{-}: \mathbf{N H}_{4}{ }^{+}\end{array}$ & MSF & MSH & MSPA & MSR & MST & R/PA \\
\hline $100: 0$ & $2,13 \mathrm{a}$ & $1,11 \mathrm{a}$ & $3,25 \mathrm{a}$ & $2,95 \mathrm{a}$ & $6,19 \mathrm{a}$ & $0,91 \mathrm{a}$ \\
$75: 25$ & $2,11 \mathrm{a}$ & $0,84 \mathrm{a}$ & $2,96 \mathrm{a}$ & $1,51 \mathrm{~b}$ & $4,47 \mathrm{a}$ & $0,50 \mathrm{~b}$ \\
$50: 50$ & $2,08 \mathrm{a}$ & $0,75 \mathrm{a}$ & $2,83 \mathrm{a}$ & $1,64 \mathrm{ab}$ & $4,47 \mathrm{a}$ & $0,54 \mathrm{ab}$ \\
$25: 75$ & $1,20 \mathrm{~b}$ & $0,31 \mathrm{~b}$ & $1,71 \mathrm{~b}$ & $0,48 \mathrm{bc}$ & $2,19 \mathrm{~b}$ & $0,27 \mathrm{~b}$ \\
$0: 100$ & $0,14 \mathrm{c}$ & $0,05 \mathrm{~b}$ & $0,19 \mathrm{c}$ & $0,04 \mathrm{c}$ & $0,24 \mathrm{~b}$ & $0,25 \mathrm{~b}$ \\
\hline $\mathrm{CV}(\%)$ & 21,79 & 36,09 & 25,01 & 56,22 & 32,44 & 43,37 \\
\hline
\end{tabular}

Médias seguidas pelas mesmas letras minúsculas nas colunas não diferem estatisticamente entre si pelo teste de Tukey a $5 \%$ de probabilidade.

No tocante à produção de massa seca da planta, SILVA et al. (2010), estudando os efeitos dos íons amônio e nitrato no desenvolvimento do girassol constataram que foi significativa aos tratamentos aplicados. $O$ tratamento com a proporção 100:0 $\left(\mathrm{NH}_{4}^{+}: \mathrm{NO}_{3}{ }^{-}\right)$foi o que apresentou menor incremento na massa seca 
nas folhas, haste e raiz das plantas.O aumento do amônio demonstra efeitos negativos, seguindo a mesma tendência observada nas variáveis de comprimento.

ANDRADE (1994) verificou que as plantas que receberam nitrato, combinado ou não com amônio, apresentaram produção de massa seca e concentração de nitrogênio total superiores aos das plantas tratadas apenas com amônio, tanto na parte aérea quanto no sistema radicular, e que a atividade da redutase do nitrato foi estimulada até a proporção de 50/50 de nitrato/amônio.

Os maiores valores médios de área foliar, foram encontrados nas proporções de nitrato:amônio de100:0 e 75:25 (Tabela 4). As médias de área foliar especifica e razão de área foliar foram maiores na proporção 0:100. Quanto às clorofilas $a, b$ e total, a proporção que favoreceu os maiores valores das médias foi a $50: 50\left(\mathrm{NO}_{3}{ }^{-}\right.$ $\left.: \mathrm{NH}_{4}^{+}\right)$.

Ao estudarem efeito do nitrato e amônio sobre o crescimento e eficiência de utilização do nitrogênio em mandioca, CRUZ et al. (2006), observaram que os tratamentos constituídos exclusivamente de nitrato ou amônio também proporcionaram menor número de folhas. Esses resultados, tomados em conjunto, explicam os menores valores de massa seca da folhas obtidos por esses tratamentos. Nas plantas supridas apenas com nitrato, verificou-se redução de aproximadamente $10 \%$ no número de folhas e de $15 \%$ na área foliar; já o amônio proporcionou reduções de $21 \%$ e $31 \%$, respectivamente.

De acordo com COSTA et al. (2008), o aumento de doses de N propicia maiores teores de clorofila e aumento na concentração desse nutriente no capimMarandu.

TABELA 4. Valores médios das variáveis área foliar (AF), área foliar específica (AFE), razão de área foliar (RAF), clorofila a, b e total (CL $a, C L$ b e CL Total) das plantas de manjericão submetidas a diferentes proporções de $\mathrm{NO}_{3}{ }^{-} \mathrm{NH}_{4}{ }^{+}$em solução nutritiva. Cruz das Almas, 2015.

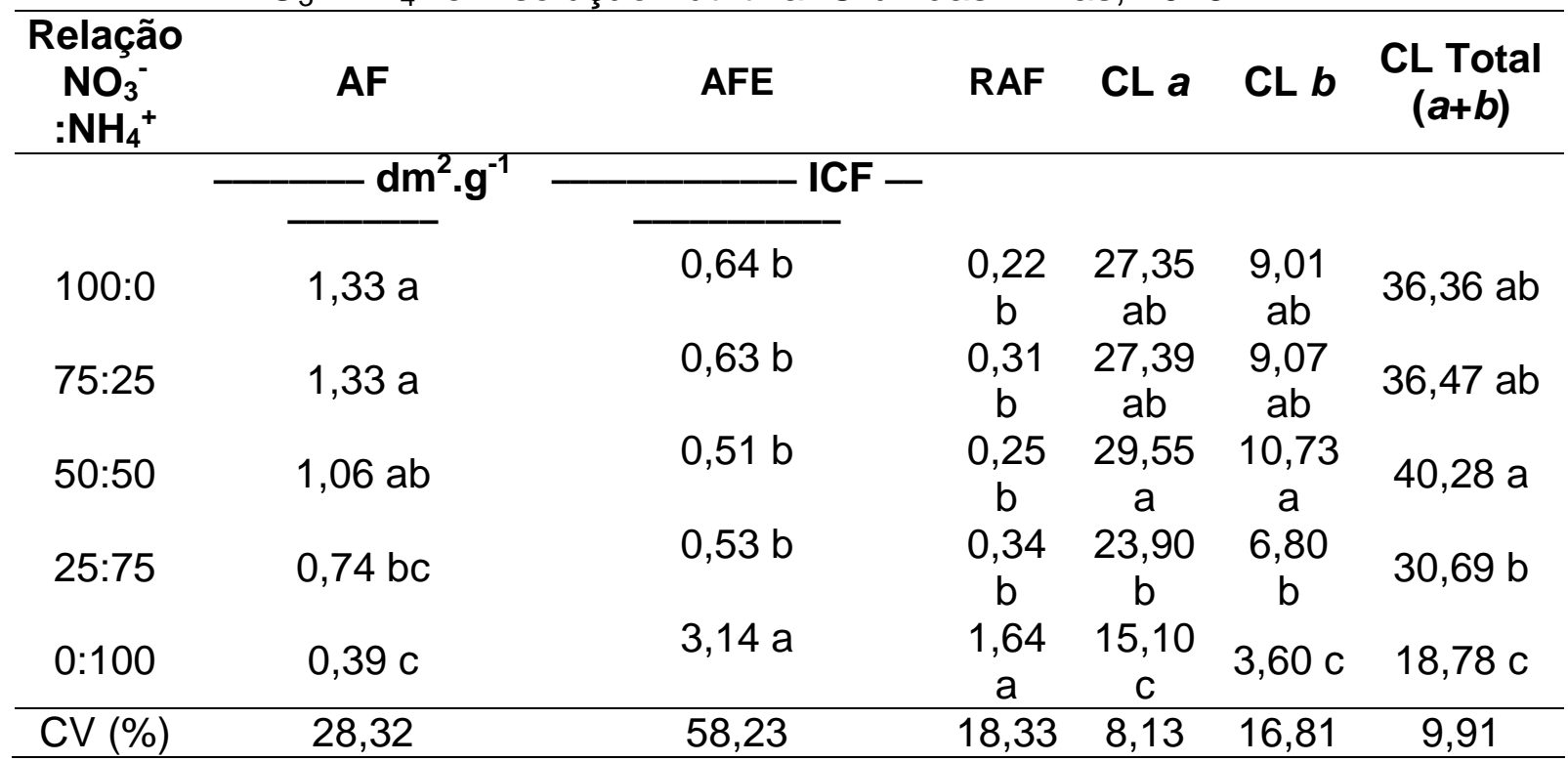

Médias seguidas pelas mesmas letras minúsculas nas colunas não diferem estatisticamente entre si pelo teste de Tukey a $5 \%$ de probabilidade

O nitrato e o amônio podem alterar os teores de clorofila, causando distúrbios à fotossíntese, como observaram RUAN et al. (2007), em Camellia sinensis. De forma contrária, GUO et al. (2012) verificaram que as fontes de $\mathrm{N}$ não alteraram os 
teores de clorofila em Catharanthus roseus. As clorofilas são responsáveis pela captação da luz no complexo antena, além de serem componentes primordiais dos centros de reação nos fotossistemas (CARNEIRO, 2015).

\section{CONCLUSÕES}

A ausência do nitrato na solução nutritiva foi prejudicial para as variáveis altura da planta, diâmetro da haste e comprimento de raiz. A variável número de folhas foi favorecida pelas proporções 100:0 e 75:25 de nitrato e amônio.

As proporções 100:0, 75:25 e 50:50 $\left(\mathrm{NO}_{3}{ }^{-} \mathrm{NH}_{4}{ }^{+}\right)$favoreceram as variáveis massa seca das folhas, hastes, parte aérea e massa seca total. $O$ maior valor de massa seca da raiz e de relação raiz/parte aérea foi observado no tratamento cuja proporção $\left(\mathrm{NO}_{3}{ }^{-}: \mathrm{NH}_{4}{ }^{+}\right)$foi 100:0. As proporções $\left(\mathrm{NO}_{3}{ }^{-}: \mathrm{NH}_{4}{ }^{+}\right)$100:0 e $75: 25$ proporcionaram as plantas maiores valores de área foliar, área foliar específica e razão de área foliar.

A proporção $\left(\mathrm{NO}_{3}{ }^{-}: \mathrm{NH}_{4}{ }^{+}\right)$50:50 foi responsável pelos maiores valores das clorofilas $a, b$ e total $(a+b)$.

\section{REFERÊNCIAS}

ALVES, A. C; JESUS, F. N; SILVA, P. C. C.; SANTOS, A. R; SOUZA, G. S. Diagnose nutricional de mudas de girassol submetidas a proporções de amônio e nitrato. Enciclopédia Biosfera, v. 9, p. 723, 2013.

ANDRADE, S. R. M. Efeito da proporção $\mathrm{NH}_{4}{ }^{+}: \mathrm{NO}_{3}{ }^{-}$na composição da fraçãonitrogenada e na atividade das enzimas de redução e assimilação de nitrogênioem plantas de capim-Colonião (Panicum maximum Jacq.). 1994. 49p. Dissertação (Mestrado) - Universidade Federal de Viçosa, Viçosa.

BLANK, Arie Fitzgerald et al. Maria Bonita: cultivar de manjericão tipo linalol. Pesq. agropec. bras., Brasília, v. 42, n. 12, p. 1811-1813, Dec. 2007.

BLANK, A. F. et al. Comportamento fenotípico e genotípico de populações de manjericão. Horticultura Brasileira, v. 28, n. 3, p. 305-310, 2010.

CRUZ, J. L.; PELACANI, C. R.; ARAUJO, W. L. Efeito do nitrato e amônio sobre o crescimento e eficiência de utilização do nitrogênio em mandioca. Bragantia, Campinas, v. 65, n. 3, p. 467-475, 2006.

COSTA, K. A. P.; FAQUIM, V.; OLIVEIRA, I. P.; ARAUJO, J. L.; RODRIGUES, R. $B$. Doses e fontes de nitrogênio em pastagem de capim-Marandu. II - Nutrição nitrogenada da planta. Revista Brasileira de Ciência do Solo, Viçosa, v. 32, p. 1601-1607, 2008.

FERREIRA, D.F. Sisvar: A computer statistical analysis system. Ciência e Agrotecnologia, Lavras, v.35, n.6, p.1039-1042, nov./dez. 2011.

GUIMARÃES, M. M. C; CAIRO, P. A. R; NEVES, O. S. C. Crescimento de Eucalyptus urophylla em Meio Hidropônico com Diferentes Proporções de Nitrato e Amônio. Floresta e Ambiente, v. 21, p. 52-61, 2014. 
GUO, S. et al. Ammonium nutrition increases photosynthesis rate under water stress at early development stage of rice (Oryza sativa L.). Plant and Soil, v. 296, p. 115-124, 2007.

CARNEIRO, M. M. L. C. et al. Fotorrespiração e metabolismo antioxidante em plantas jovens de seringueira cultivadas sob diferentes fontes de nitrogênio (NO3 - e NH4 +). Revista Brasileira de Ciências Agrárias, v. 10, n. 1, 2015, p. 66-73. Universidade Federal Rural de Pernambuco. Pernambuco, Brasil.

RIBEIRO, M. O. et al. Efeito das interações entre os íons amônio e nitrato na fisiologia do crescimento do amendoinzeiro. Rev. Ceres, v.59 n.5. Viçosa Sept./Oct. 2012.

RUAN, J. et al. Effect of nitrogen form and root-zone ph on growth and nitrogen uptake of tea (Camellia sinensis) plants. Annals of Botany, v. 99, p. 301-310, 2007.

SILVA, P. C. C, COUTO, J. L, SANTOS, A. R. Efeito de íons amônio e nitrato no desenvolvimento do girassol em solução nutritiva. Revista da FZVA, v. 17, n. 1, p. 104-114, 2010.

SOUSA, V. F. L.;. OLIVEIRA, F. A .; OLIVEIRA, F. R. A.; CAMPOS, M. S.; MEDEIROS, J. F. Efeito do Nitrato e Amônio Sobre o Crescimento da Berinjela. Revista Verde (Mossoró - RN - Brasil) v.5, n.3, p. 80 - 88 julho/setembro de 2010.

TEIXEIRA, J.P.F.; MARQUES, M.O.M.; FURLANI, P.R.; FACANALLI, R. Essential oil contents in two cultivars of basil cultivated on NFT-hydroponics. IN: Proceedings of the First Latin-American Symposium on the Production of Medicinal, Aromatic and Condiments Plants, Acta Horticulturae, v.569, p.203208, 2002. 